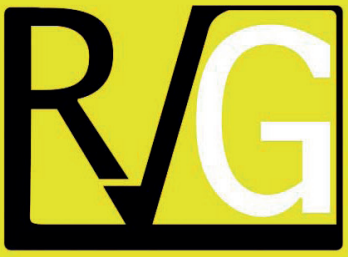

Julio - Septiembre, 2021

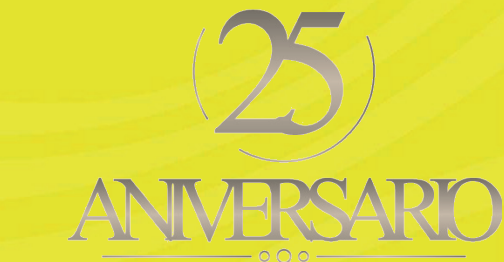

ANMERSARO
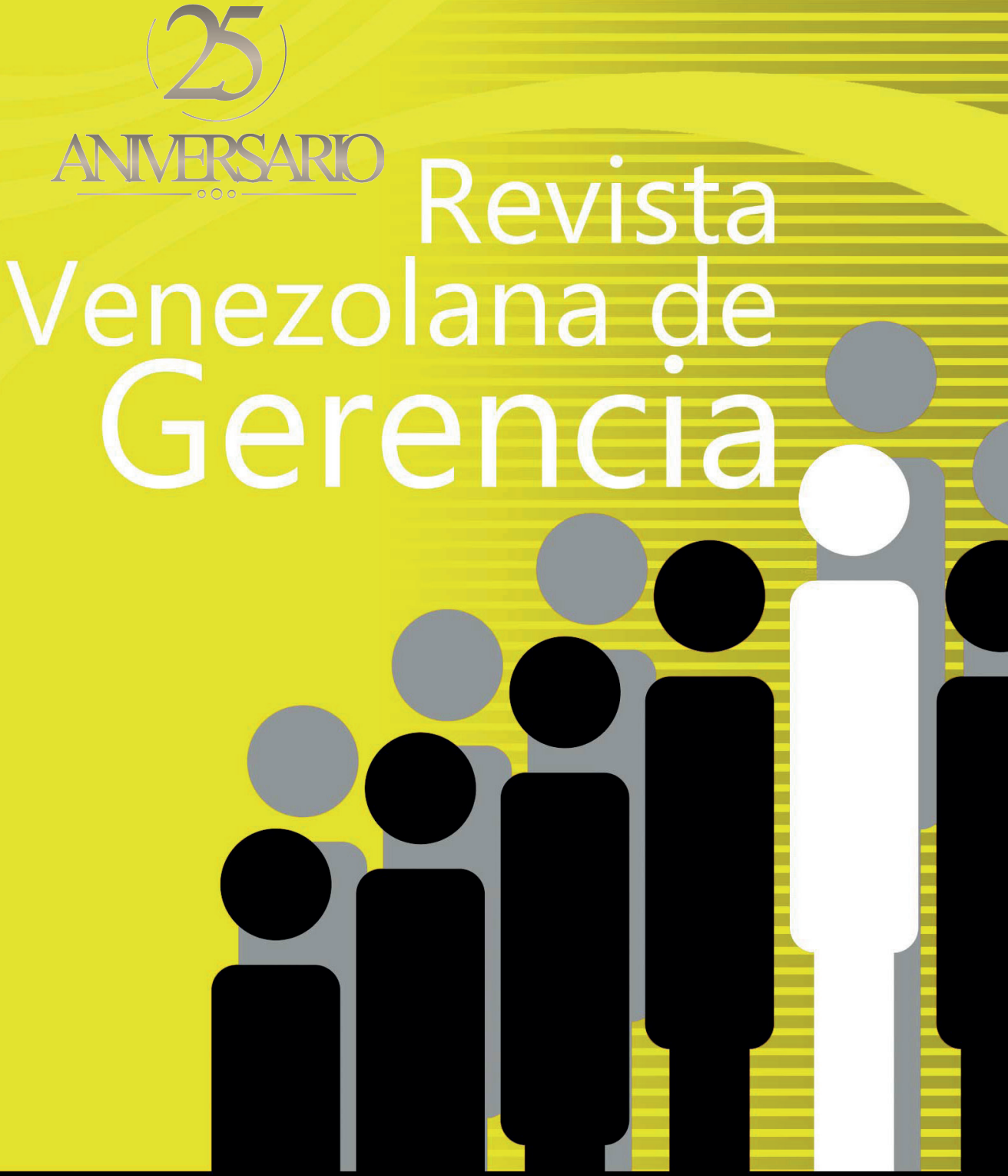

UNIVERSIDAD DEL ZULIA (LUZ)

Facultad de Ciencias Económicas y Sociales Centro de Estudios de la Empresa

ISSN 1315-99

Esta obra está bajo una licencia de Creative Comm Reconocimiento-NoComercial-Compartirlgual 3.0 Unpo http://creativecommons.org/licenses/by-nc-sa/3.0/deed.es 
COMO CITAR: Gallardo Canales, R., Pinto Inostroza, M., y Aguirre Boza, Á. (2021). Desempeño Académico de Estudiantes Universitarios con Ayudas Financieras: Una Revisión de Literatura. Revista Venezolana de Gerencia (RVG), 26(95), 683$704 . \quad$ https://doi.org/10.52080/
Universidad del Zulia (LUZ)

Revista Venezolana de Gerencia (RVG)

Año 26 No. 95 Julio-Septiembre 2021, 683-704

ISSN 1315-9984 / e-ISSN 2477-9423

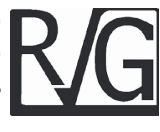

\title{
Desempeño Académico de Estudiantes Universitarios con Ayudas Financieras: Una Revisión de Literatura*
}

\author{
Gallardo Canales, Rodrigo** \\ Pinto Inostroza, Mario*** \\ Aguirre Boza, Álvaro****
}

\section{Resumen}

En los últimos años se ha observado un incremento significativo en la matrícula de estudiantes de menores ingresos a la universidad, dado el mayor financiamiento a través de becas y préstamos; sin embargo, la efectividad de estos programas no es tan clara considerando las bajas tasas de retención, persistencia y graduación. El presente estudio tiene como propósito identificar los efectos de las ayudas financieras sobre los indicadores de logros estudiantiles a través de una revisión sistemática de literatura internacional entre 2015 y 2020 para encontrar relaciones entre el desempeño de los estudiantes y las ayudas financieras que reciben. Los resultados demuestran que, en el caso de las becas, el efecto es positivo en los indicadores de rendimiento y graduación; empero, en el caso de los préstamos, el desempeño es heterogéneo y denota un estrés financiero para los estudiantes. Se concluye la necesidad de investigaciones futuras que determinen de manera consistente la significancia de los préstamos en los resultados académicos dada la gran inversión pública en este ámbito, y la vinculación del perfil de

Recibido: 20.03 .21

Aceptado: 10.05 .21

* $\quad$ Apoyado por beca de investigación 2020 de la Facultad Tecnológica de la Universidad de Santiago de Chile

** Magíster en Administración y Dirección de Empresas MBA, Ingeniero Comercial y Licenciado en Ciencias de la Administración, Universidad de Santiago, Chile. Profesor Asistente, Departamento de Tecnologías de Gestión, Facultad Tecnológica, Universidad de Santiago, Chile. E-mail: rodrigo.gallardo@usach.cl ORCID: https://orcid.org/0000-0001-9774-3916

*** Magíster en Seguridad Industrial, Universidad Politécnica de Cataluña, Barcelona, España. Ingeniero Ambiental DuocUC, Chile. Profesor Asistente, Departamento de Tecnologías de Gestión, Facultad Tecnológica, Universidad de Santiago, Chile. E-mail: mario.pinto.i@usach.cl ORCID: https://orcid.org/00000003-1274-3082

**** Magíster en arquitectura de la Pontificia Universidad Católica de Chile, Arquitecto, licenciado en arquitectura Universidad Mayor. Profesor Asistente, Departamento de Tecnologías de Gestión, Facultad Tecnológica, Universidad de Santiago, Chile. E-mail: alvaro.aguirre@usach.cl ORCID: https://orcid.org/0000-0002-7392$\underline{9692}$ 
Gallardo Canales, Rodrigo; Pinto Inostroza, Mario; Aguirre Boza, Álvaro

Desempeño Académico de Estudiantes Universitarios con Ayudas Financieras: Una

Revisión de Literatura

estudiantes e instituciones con el impacto de los programas de financiamiento, para contribuir a la selección de programas efectivos y la toma de decisiones de la política pública.

Palabras clave: Desempeño Académico; Estudiante Universitario; Ayuda Financiera; Políticas Públicas.

\title{
Academic Performance of College Students Awarded with Financial Aid: A Literature Review
}

\begin{abstract}
In recent years, a significant increase of limited resources students attending university has been observed, as a response to higher financial aid through scholarships and loans. Nevertheless, it is unclear their effectiveness considering the withholding rates, persistency, and graduation. The present study has taken as an objective to identify the effects of financial aid on student achievement indicators through a systematic international literature revision from 2015 to 2020 to establish relations between student performance and financial aid. Results demonstrate that, in the case of scholarships, the effect is positive within the ambits of performance and graduation; by comparison, loans' effects are heterogeneous and show a financial stress for students. To conclude, the necessity of future investigations is clear in order to consistently determine loans' significance related to academic results because of the large investment in this field, as well as the relation between students' profile and academic institutions regarding the impact of funding programs; in order to contribute to the selection of effective programs and public policy decision-making.
\end{abstract}

Keywords: Academic performance; University Student; Public Policies; Financial Aid.

\section{Introducción}

En las últimas décadas, la incorporación de una dimensión social en la educación superior se ha constituido como un factor relevante en las políticas universitarias, transitando hacia la consolidación de instituciones que promuevan la inclusión, el acceso y la equidad, pero además con el foco en la excelencia (Catalán \& Santelices, 2014). En este contexto, el compromiso con la justicia social ha trascendido como un fenómeno global a partir de la ampliación de la participación de grupos subrepresentados para distribuir 
los beneficios de la educación superior y disminuir las desigualdades (Young et al. 2013), lo que se ha traducido en una ampliación dramática de la participación en educación superior, donde la población entre 18 y 24 años en la educación superior se duplicó en América Latina (von Hippel \& Hofflinger, 2020).

Sin embargo, en los últimos tiempos se ha debatido la forma de financiar los estudios superiores y sus efectos en factores tales como el desempeño académico y los índices de graduación y deserción (Berlanga et al. 2017). Por ello surgen aprensiones, tanto desde los gobiernos como las comunidades educativas y la sociedad civil para poner atención a los mecanismos de financiamiento y sus repercusiones para mejorar no solo la participación de grupos subrepresentados sino también su desempeño académico y el cumplimiento de su educación superior (Considine \& Zappala, 2002; Correa, 2018).

Sobre lo anteriormente expuesto, el presente artículo busca identificar los efectos de las ayudas financieras sobre los indicadores de logros estudiantiles a través de una revisión sistemática de literatura internacional de los últimos cinco años para proponer mejoras en las políticas públicas de programas que ofrecen ayudas, con la intención de otorgar un aporte tanto social, desde la perspectiva de adecuar la inserción a la educación superior de grupos subrepresentados e impulsar la inclusión; y, un aporte científico, desde la perspectiva de equipar a la mayor cantidad de la población con herramientas de conocimiento para mejorar su comunidad, a través de su avance académico.

Desde la mirada de las instituciones de educación superior, ha sido muy complejo retener a los estudiantes y completar el ciclo con la obtención del certificado de título y/o grado. Tinto (1993) percibe esta dificultad como una inversión desaprovechada o perdida para la universidad y para el estudiante.

La investigación reciente sobre esto ha sido concluyente, siendo los factores económicos uno de los principales predictores de acceso y elección de estudios (Troiano y Elias, 2014), obteniendo hallazgos sobre la relación entre el financiamiento y el rendimiento académico en el proceso de enseñanza-aprendizaje. Por esta razón, es necesario identificar cuáles son los efectos del desempeño académico en los estudiantes universitarios beneficiados de ayudas financieras, según la literatura internacional de los últimos cinco años, y analizar su impacto sobre los principales indicadores de éxito de su proceso formativo.

\section{Desempeño Académico y Ayudas Financieras: aspectos metodológicos}

La revisión de la literatura se realizó mediante una búsqueda sistemática de los últimos cinco años, entre 2015 y 2021, en la colección principal de la base de datos Web of Science (WoS), Scopus y Scielo por tratarse de algunos de los repositorios con mayor relevancia internacional.

La búsqueda se realizó a partir de un listado de palabras clave y fue focalizada en artículos de revistas de diversas áreas disciplinares. Los términos de la búsqueda, con sus respectivos booleanos de extensión para rastrear categorías desde sus raíces se indican en la Tabla 1. 


\section{Tabla 1}

\section{Términos de Búsqueda para la Recopilación de Literatura}

\begin{tabular}{ll}
\hline Ámbito & Términos de búsqueda \\
\hline \multirow{2}{*}{ Desempeño Académico } & $\begin{array}{l}\text { Academic Performance, Academic Result, GPA (Grade Point } \\
\text { Average), Grades, Grade Point Average, Approbation Rates, } \\
\text { Student Success* }\end{array}$ \\
\hline
\end{tabular}

Nota. *Desempeño Académico, Resultado Académico, GPA (Grade Point Average), Calificaciones, Promedio de Calificaciones, Índices de Aprobación, Éxito estudiantil.

${ }^{* *}$ Ayuda Financiera, Préstamo, Beca Académica, Beca.

*** Educación Superior, Educación Terciaria, Educación Postsecundaria, Instituto Profesional, Universidad, Pregrado.

Fuente: elaboración propia.

De la búsqueda de literatura se obtuvieron 237 artículos para las siguientes categorías: "Investigación en Educación", "Economía", "Educación de Disciplinas Científicas", "Ciencias de la Información", "Medicina General Interna”, "Ciencias Sociales Interdisciplinarias", "Sociología", "Medio Ambiente y Salud Ocupacional”,
"Ciencias Multidisciplinarias" y "Educación Especial". De esta muestra se excluyeron 141 artículos por factores de temas que se escapan al objetivo de esta revisión de literatura y factores de formalidades que impiden un análisis completo del artículo. Esos factores están detallados en la Tabla 2.

\section{Tabla 2}

Factores excluyentes para la revisión de literatura

\begin{tabular}{llc}
\hline & \multicolumn{1}{c}{ Factor Excluyente } & $\begin{array}{c}\text { Cantidad de Artículos Ex- } \\
\text { cluidos }\end{array}$ \\
\hline Según formalidades & $\begin{array}{l}\text { El artículo no está en inglés o } \\
\text { español }\end{array}$ & 5 \\
\hline El artículo es de naturaleza teórica & 8 \\
\hline \multirow{2}{*}{ Según temática } & $\begin{array}{l}\text { Educación Secundaria, Primaria o } \\
\text { Posgrado }\end{array}$ & 26 \\
\hline
\end{tabular}




\section{Cont... Tabla 2}

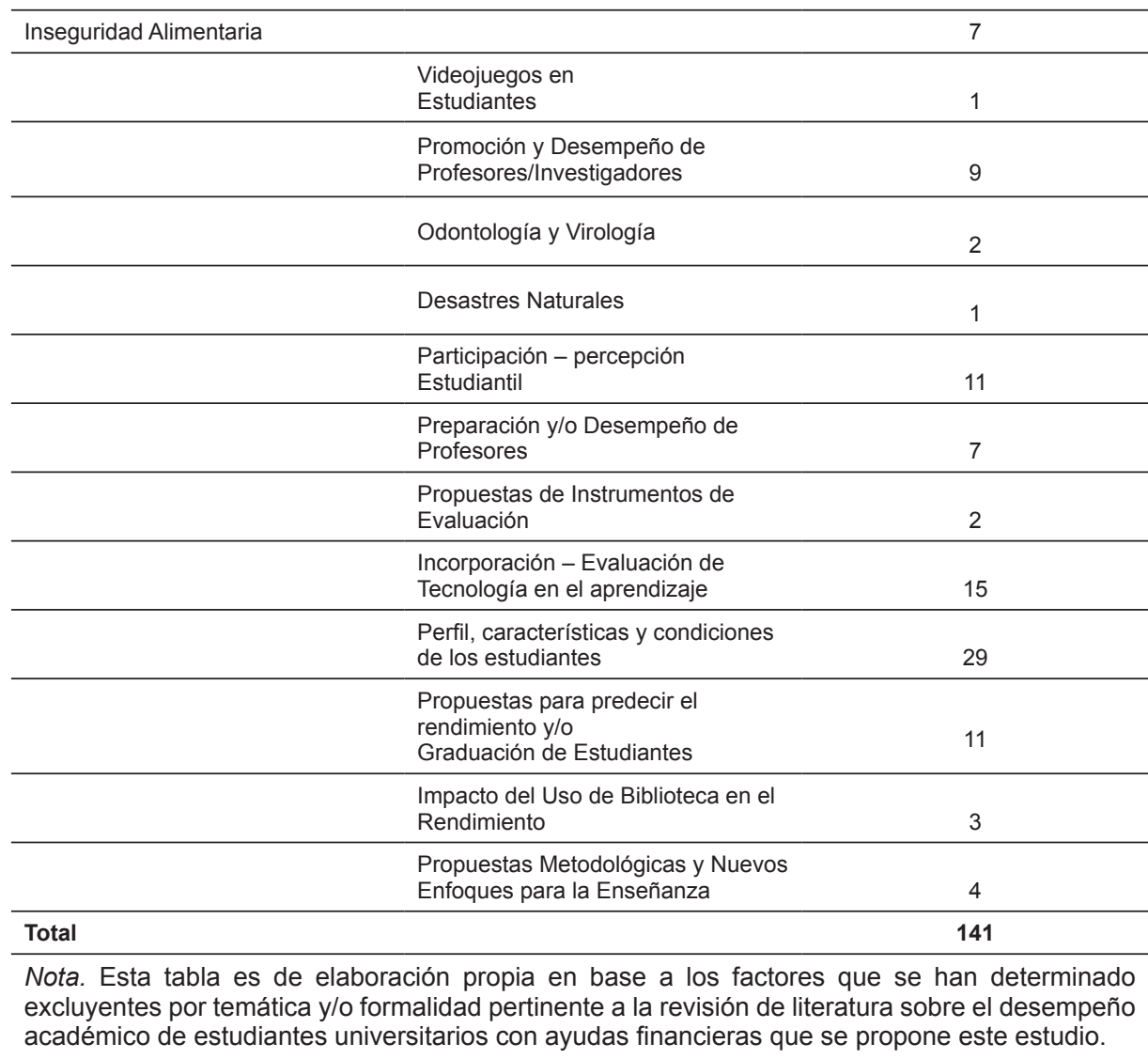

De esta forma, considerando los artículos recopilados que quedan excluidos de la revisión, por factores no pertinentes a este estudio, se aceptaron 70 artículos en total que cumplen con los siguientes requisitos: pertenecer a una investigación publicada entre 2016 y 2021, en una publicación de indexación WoS, Scopus o Scielo, que responda a los términos de búsqueda relativos a desempeño académico, ayuda financiera y estudiante universitario, y sus respectivos booleanos de extensión. 


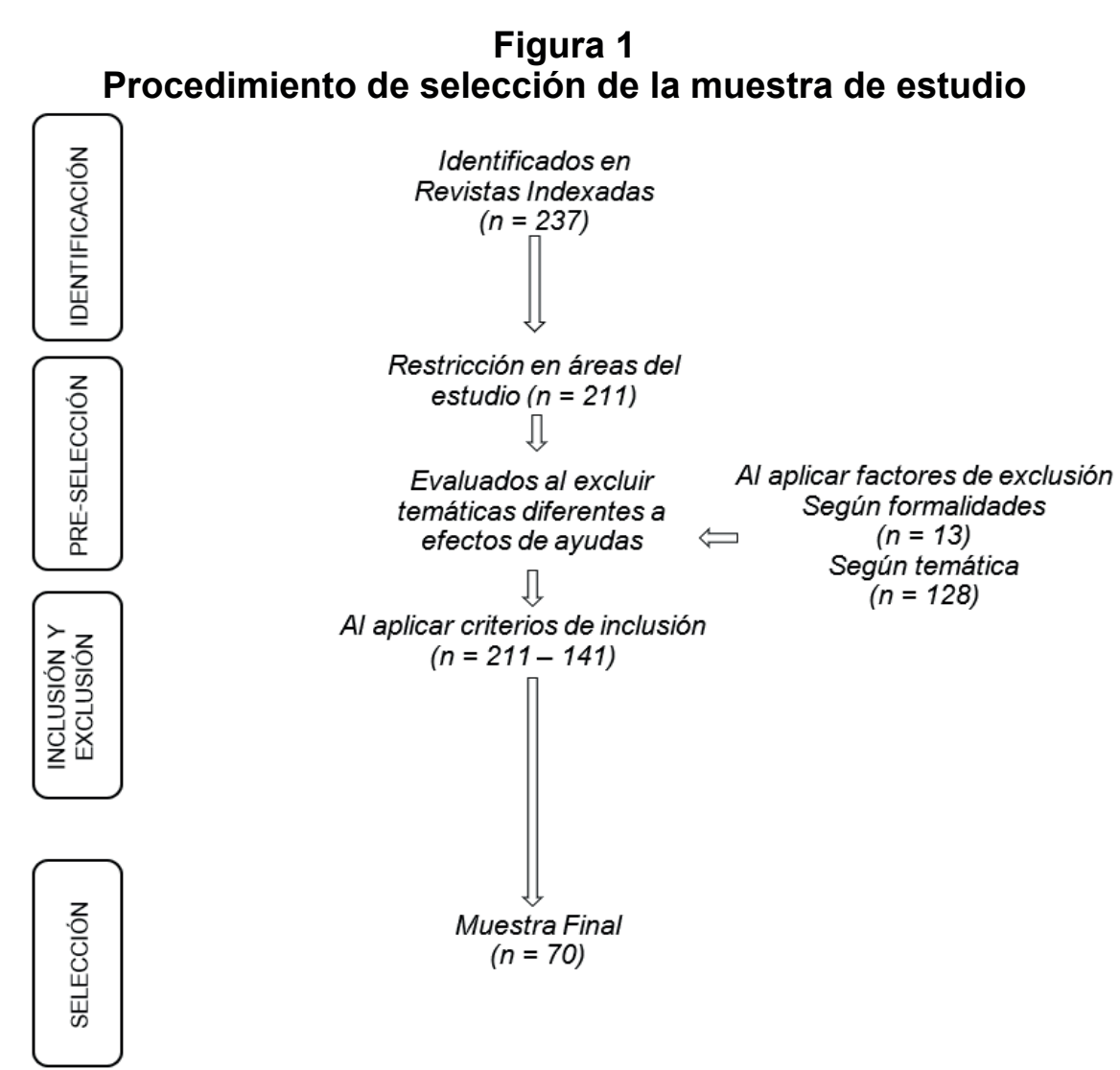

Fuente: elaboración propia sobre la selección de la muestra de este estudio.

La muestra final de 70 artículos derivados de la revisión sistemática fue organizada a partir de los efectos en el desempeño académico de estudiantes universitarios con ayudas financieras, a través de una mirada transversal, debido a la amplitud y diversidad de áreas disciplinares. El análisis de la información se realizó a partir de la relación del tipo de ayudas financieras, de naturaleza de beca o préstamo, condicionado en base a mérito o necesidad, con indicadores de desempeño y/o éxito académico como la compleción, persistencia, calificaciones y tiempo de graduación de los estudiantes durante su periodo de estudios en la universidad. El corpus completo se puede apreciar en detalle de año, autor, título y palabras claves. 


\section{Caracterización de la literatura sobre Desempeño académico con ayuda financiera}

El propósito de la revisión de literatura reciente sobre la relación entre el desempeño académico y la ayuda financiera se justifica en la necesidad de identificar los logros y los desafíos de becas y préstamos o créditos disponibles para los y las estudiantes en la educación superior, dado por indicadores de éxito académico. Esta investigación funcionará como un punto de partida para estudios posteriores sobre la adecuación de políticas públicas que faciliten ayudas financieras más efectivas para estudiantes universitarios.

Es posible realizar un primer acercamiento a estos textos a partir de sus conceptos claves, para luego indagar en factores externos: geografía, es decir, el país sobre el cual se realizan las investigaciones y tiempo, entendido como el año en el cual se publica la investigación; para luego explorar factores internos: la categorización de los artículos según enfoque.

Con ello en mente, los conceptos claves de la literatura seleccionada se pueden visualizar en la nube de palabras generada a partir de los títulos y palabras claves de cada artículo en la Figura 2.

\section{Figura 2 \\ Nube de Palabras Sobre el Desempeño Académico con Ayudas Financieras}

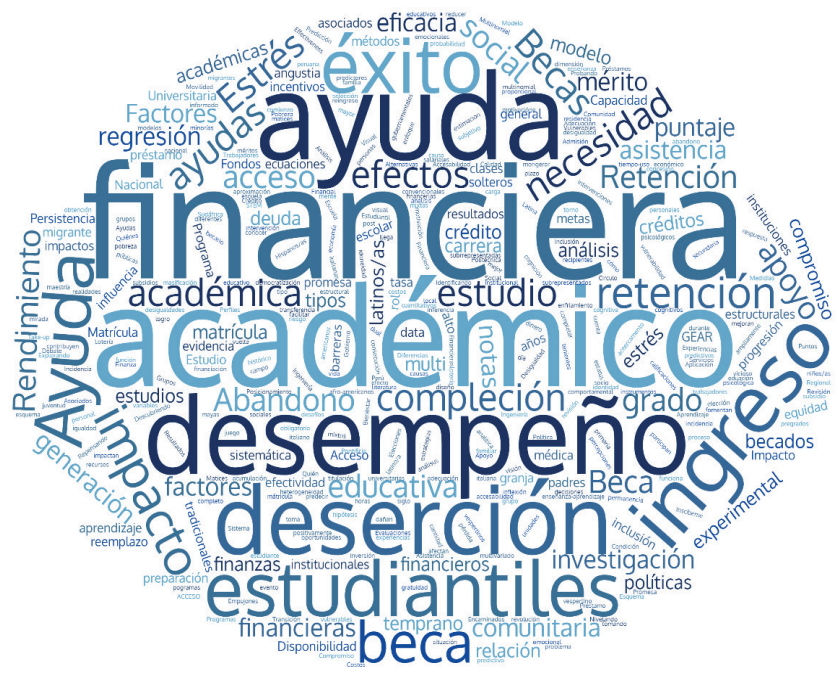

Nota. Figura de elaboración propia sobre los conceptos claves en base a títulos y palabras claves de los artículos seleccionados. En la generación de esta nube de conceptos, se excluyeron palabras comunes como conectores, hilativos y pronombres.

Fuente: Elaboracion propia 
La Figura 2 presenta una alta incidencia de los conceptos de la deserción, el impacto, el estrés y la necesidad de los y las estudiantes en los artículos, lo cual implica una preocupación hacia estos factores a la cual las ayudas financieras buscan responder. Surgen, también de forma marcada, los conceptos de abandono, compleción, mérito y éxito, evidenciando impresiones mixtas en torno a la relación entre la ayuda financiera y el desempeño académico.

\section{Factores externos: Elementos de país y año de publicación}

Dentro de los factores externos que condicionan las investigaciones revisadas, es importante categorizar el país sobre el cual se realiza el estudio y el año en el cual fue publicada dicha investigación para analizar el impacto de las ayudas financieras sobre el desempeño estudiantil en determinados espacios y tiempos, (gráfico 1).

\section{Gráfico 1 \\ Revisión de Literatura Según País de Investigación}

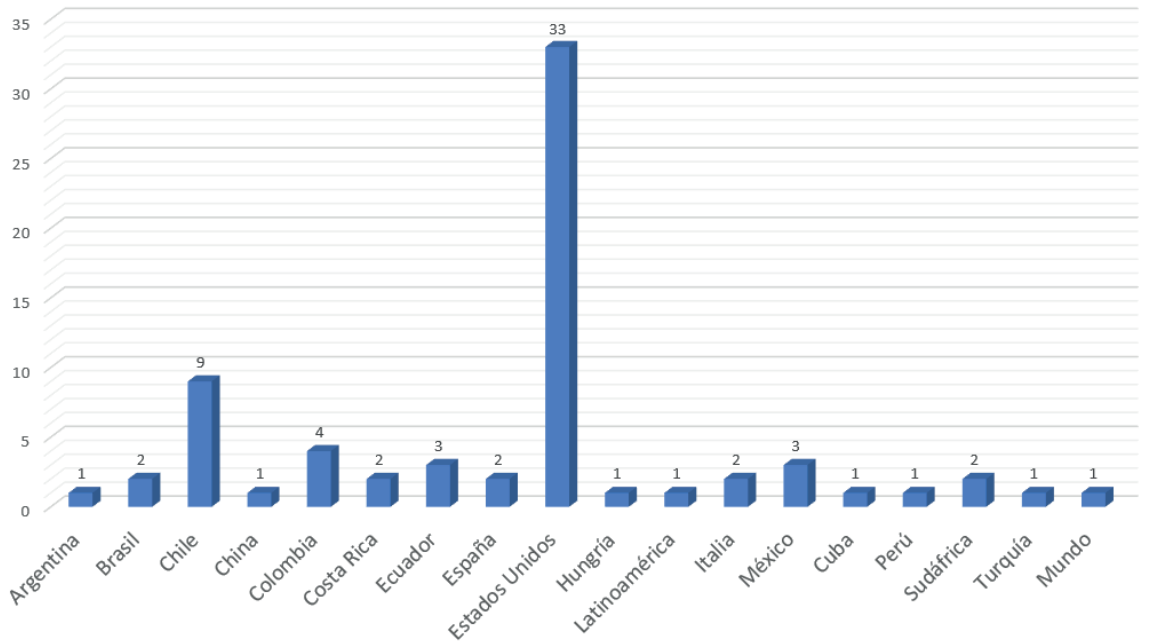

Fuente: elaboración propia ${ }^{1}$

1 Este gráfico es de elaboración propia, denotando la cantidad de publicaciones incorporadas en la muestra de esta investigación según país. Dentro de esta selección, existen también dos artículos que incluyen datos empíricos de más de un país (Paz Maldonado, 2020; Lattuada, 2017) correspondientes a un estudio de la región latinoamericana y un estudio a nivel mundo, respectivamente. 
En mayor detalle, en la Figura 3 se aprecia que los artículos seleccionados denotan un gran interés por la comprensión de las ayudas financieras y su efecto sobre el desempeño académico en Estados Unidos, ya que existe una categorización especial en dicho país que presenta opciones de ayudas financieras tanto según espacio físico, entendidos como estados (Jones, et al. 2020; Bettinger, et al. 2019); como por condiciones económicas, a modo de ingreso (Ononye y Bong, 2018; Roksa y Kinsley, 2019; Gershenfeld, Zhan y Hood, 2019); y, etnicidad, a modo de grupos sociales minoritarios (Mendez y Bauman, 2018; Gipson, Mitchell y McLean, 2018). Por otra parte, en
Latinoamérica, los estudios son menores y la investigación al respecto está empezando a indagar no solo en ayudas financieras generadas para estudiantes de bajos ingresos (Melguizo, Sanchez y Velasco, 2016) sino, además, dirigidas hacia minorías indígenas y estudiantes en situación de discapacidad (Paz, 2020; Angarita, Solano y Contreras, 2017).

Por otro lado, la selección de artículos para este estudio se limita a los años 2015 a 2021, para obtener un enfoque actual. Los años más productivos en cuanto a investigación sobre la relación entre las ayudas financieras y el desempeño académico se reflejan en el Gráfico 2.

\section{Gráfico 2 \\ Revisión de Literatura Según Año de Publicación}

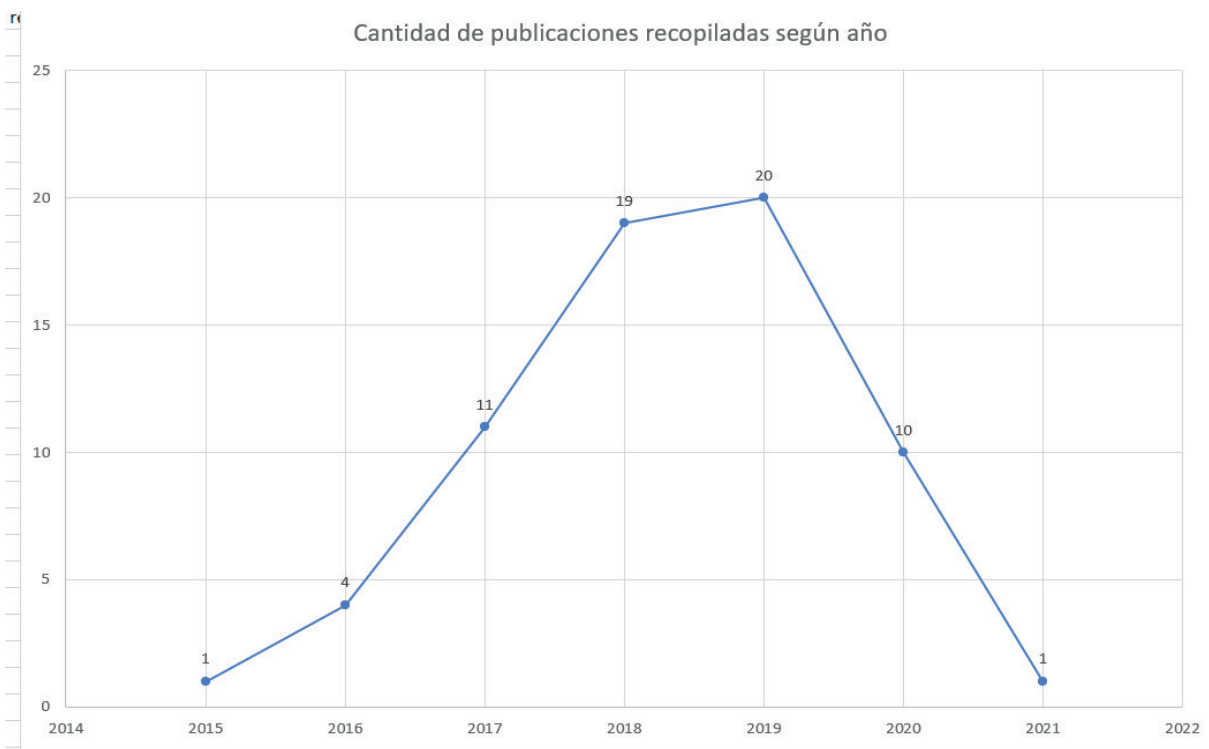

Fuente: elaboración propia 
El gráfico 4 evidencia la cantidad de artículos publicados, según año, sobre ayudas financieras y desempeño académico recopilado para este estudio entre los años de 2015 a 2021, denota que la mayoría de los artículos seleccionados fueron publicados en el año 2018 y 2019; mientras que, solo hay un total de seis artículos recopilados entre 2015, 2016 y 2021. Hay 21 artículo seleccionados de los años 2017 y 2020 , asegurando que la investigación se mantenga lo más actualizada posible.

\section{Factores Internos: Indicadores de Desempeño Académico}

El análisis del corpus evidencia que las principales fuentes de financiamiento académico de estudiantes universitarios para costear sus estudios están basadas en becas y préstamos, que se obtienen por necesidad o mérito, y estas tienen un efecto sobre el desempeño académico que logran en su proceso formativo que se reflejan en términos generales de graduación, deserción, rendimiento, calificaciones, persistencia, entre otros.

Para comenzar, los resultados de Graziosi, et al. (2020) sobre el efecto de las subvenciones en el rendimiento estudiantil de 1.357 estudiantes tratados con becas y 6.058 estudiantes del grupo de control pertenecientes a cinco universidades italianas durante cuatro años, revelaron que existe una relación entre el rendimiento y recibir una subvención que podría derivar en impactos positivos en los indicadores de desempeño y éxito estudiantil.

Este resultado es compartido por Salinas, Hernández y BarbosaPalomino (2017), quienes reafirman cierta correlación entre estudiantes beneficiarios de un programa de becas en Perú y el logro académico, detectando un impacto positivo en el desempeño a partir de una muestra de 1262 alumnos, de los cuales el $16 \%$ fueron becarios. Del mismo modo, existe una asociación entre el rendimiento y persistencia de estudiantes becados en la universidad y otras variables como las calificaciones y las horas de estudio (Rodriguez, et al. 2019). Otros estudios sugieren que la recepción de una beca en conjunto con la inscripción a tiempo completo y la integración académica influencian la persistencia de estudiantes universitarios hispanos/latinos (Carales, 2020).

En el caso de alumnos que son padres solteros, las ayudas financieras junto a otras variables como los ingresos y el monto de la contribución familiar podrían ser predictores significativos del progreso hacia la finalización de estudios (Carpenter, et al. 2018).

\section{Impacto de las Ayudas Financieras en el Éxito Estudiantil}

Según esta revisión, las becas tendrían un impacto mayoritariamente positivo mientras que el efecto de los préstamos parecierasernegativo, aunque estos últimos resultados no se pueden establecer como concluyentes, ya que los resultados disponibles hasta el año 2020 sobre este factor en particular son heterogéneas debido a sus factores de desempeño y las variables relacionadas que han surgido en esta investigación. En el gráfico 3 se puede apreciar la revisión de los artículos seleccionados categorizados por enfoque principal, en cuanto a ayudas financieras a modo de becas o programas de mérito; 
préstamos y créditos universitarios; factores de desempeño, como calificaciones, graduación, deserción, y variables relacionadas como apoyo familiar y horas dedicadas el estudio; e inclusión, a modo de artículos dedicados exclusivamente a estudiantes de bajos ingresos $\mathrm{y} / \mathrm{o}$ pertenecientes a minorías subrepresentadas y/o en situación de discapacidad.

\section{Gráfico 3 \\ Categorización de artículos según enfoque principal}

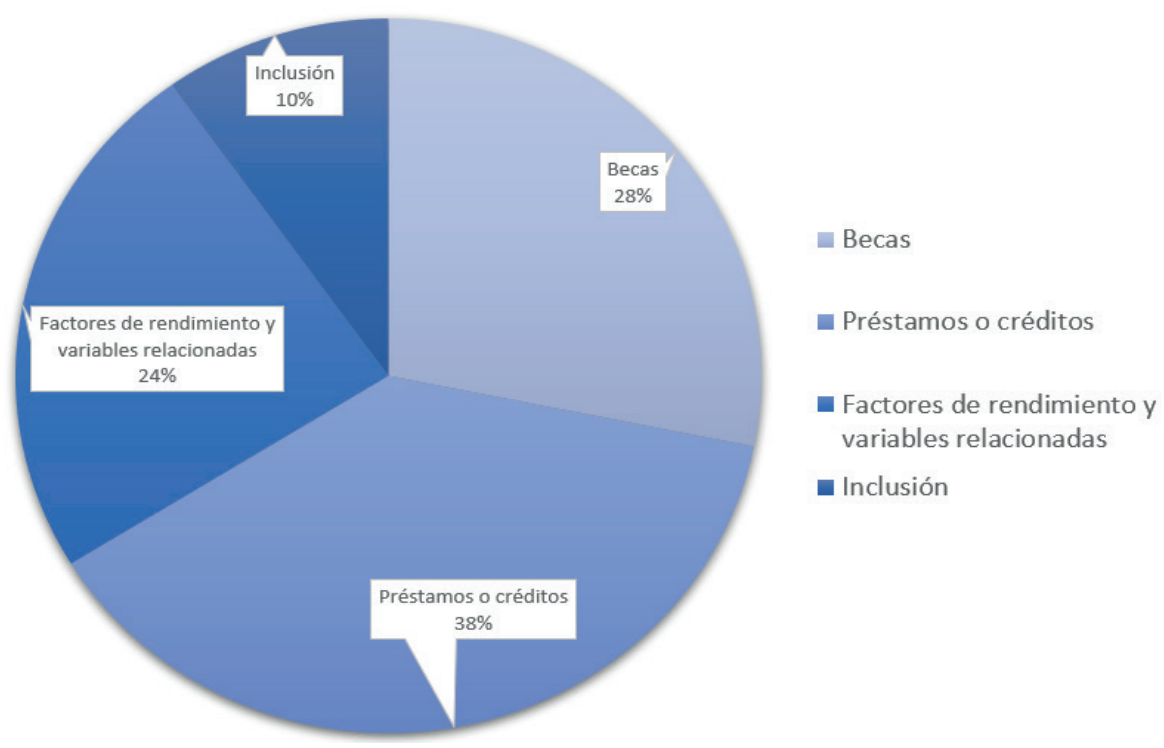

Fuente: elaboración propia

Entre las relaciones positivas entre becas y rendimiento, Graziosi et al. (2020) constatan que, entre 7.415 estudiantes de primer año matriculados en cinco universidades italianas, las subvenciones basadas en la necesidad tienen un impacto positivo y significativo en el éxito estudiantil, contribuyendo a completar los estudios, graduarse y disminuir probabilidad de abandono en primer año, principalmente en disciplinas de humanidades y ciencias sociales.
Este resultado es más heterogéneo en las áreas de ciencias, exceptuando medicina donde no se aprecia un efecto significativo (Moreira et al. 2019).

Aunado a ello, pareciera que las becas podrían aumentar la probabilidad de persistir y de graduarse (Carales, 2020), y en el caso de las ayudas orientadas a estudiantes que son padres solteros el efecto en los indicadores de desempeño académico es aún más significativo, particularmente en 
la obtención de créditos académicos, calificaciones y finalización de grado en donde la ayuda financiera podría ser un predictor significativo del progreso hacia la finalización (Carpenter et al. 2018).

Sin embargo, estudios de Goldhaber et al. (2019), se enfocan sobre programas de mérito que exigen a los estudiantes el compromiso de cumplir con las expectativas de universidades específicas y concluyen que el interés de estudiantes por estos programas ha disminuido, y podrían ser más exitosos de no exigir al estudiante un compromiso o promesa con antelación.

Asimismo, los resultados de Agasisti y Murtinu (2016) sugieren un efecto positivo para la obtención de créditos académicos en estudiantes con ayudas financieras basadas en la necesidad (Agasisti y Murtinu, 2016; Whatley y Clayton, 2020; Huang, Li, Liao y $\mathrm{Hu}, 2018$; Herbaut y Geven, 2019). La magnitud del efecto pareciera ser mayor en áreas de ingeniería que de arquitectura y diseño (Agasisti y Murtinu, 2016).

Del mismo modo, diversos autores coinciden en que el rendimiento académico muestra un GPA mayor para alumnos becados (Doyle et al. 2019; Broton, 2019; Berlanga et al. 2017; Herbaut y Geven, 2019; Berlanga et al. 2016; Coria \& Hoffman, 2016). Dicho impacto, también se presenta en otros datos e indicadores de desempeño académico como puntaje de aprobación de cursos mayor al mínimo exigido (Salinas et al. 2017); tasas de graduación más altas junto con una persistencia similar a estudiantes sin becas (Berlanga et al. 2017); y, tasas de aprobación de exámenes y de cursos también más altas (Berlanga et al. 2016).

Si bien, se logra detectar un impacto aparente de las ayudas financieras en el desempeño de los estudiantes, los resultados de Coria \& Hoffman (2016) sobre una muestra de 11956 estudiantes de una universidad de Los Ángeles en Estados Unidos, indican que los efectos sobre el rendimiento académico son estadísticamente significativos pero de tamaño pequeño, y, aparentemente, ante un cambio en el monto de la ayuda también lo hace el GPA, lo que supone una relación directamente proporcional, superando incluso a alumnos sin beneficios.

Por otra parte, las ayudas financieras basadas en préstamos tienden a mostrar una heterogeneidad en el efecto que producen en el desempeño académico de los estudiantes, a partir de la naturaleza de los programas de financiamiento estudiantil. Según los resultados de Melguizo, Sanchez y Velasco (2016), es posible constatar a partir de una muestra de 46418 estudiantes colombianos participantes de un programa de préstamos subsidiados a nivel nacional, que estos mecanismos de financiamiento estudiantil, que permiten incrementar el acceso y matrícula de alumnos de bajos ingresos, podrían aumentar la tasa de aprobación de cursos y ayudarían a mejorar el rendimiento académico. Este resultado es compartido por Marx y Turner (2019) que sugieren un aumento significativo del rendimiento medido a través de GPA y de los créditos académicos intentados/ obtenidos de estudiantes con préstamos.

En contraste, los resultados de Stoddard, Urban y Schmeiser (2018) revelan que el GPA de alumnos con préstamos de dos grandes universidades de Montana en Estados Unidos es levemente menor que el grupo de control (sin préstamo) y que este impacto negativo sobre el rendimiento tiene una magnitud mayor en los primeros años 
sobre el GPA, la obtención de créditos académicos y la persistencia.

Otros autores del corpus sugieren una posible asociación entre una mayor cantidad de préstamos y la disminución de calificaciones y de probabilidad de graduación, donde aparentemente la acumulación de deuda de préstamos estudiantiles de primer año podría ser un predictor del efecto negativo en las calificaciones (Destin \& Svoboda, 2018).

A ello se suman otros estudios que destacan el impacto negativo del estrés financiero sobre la autoeficacia del estudiante y su decisión al escoger una profesión (Robb, 2017; Britt et al. 2017). Esto se apoya en el estudio de Cadaret y Bennett (2019) que establece una directa relación entre el estrés financiero, causado por préstamos y créditos universitarios, y la angustia psicológica.

En resumen, las ayudas financieras a partir de becas $y$ préstamos tienen un impacto en los resultados académicos de estudiantes universitarios; particularmente, aquellas ayudas basadas en la necesidad tienen un impacto positivo con una magnitud del efecto que depende de otras variables relacionadas como la caracterización de las instituciones y estudiantes junto a las variables de contexto. Dicha magnitud podría cambiar en función del monto de la ayuda.

Según Ononye y Bong (2018), las áreas en que los efectos serían más significativos son las disciplinas de ingeniería y algunas especialidades CTIM (Ciencia, Tecnología, Ingeniería y Matemáticas) o STEM (por sus siglas en inglés: Science, Technology, Engineering and Mathematics). Por otra parte, los préstamos estudiantiles tienen un efecto más heterogéneo en el rendimiento de los estudiantes, donde pareciera que aquellos tipos de préstamos más tradicionales tendrían un efecto negativo y aquellos diseñados en programas más flexibles tendrían un impacto positivo. En este ámbito se ubican estudios como Chen y Hossler (2017) que evidencian el éxito de ayudas financieras para estudiantes que buscan retomar sus estudios universitarios o han demorado más del tiempo tradicional en completar sus estudios.

\subsection{Ayudas financieras y tasas de graduación y deserción}

Respecto a la vinculación entre las ayudas financieras y la graduación de los estudiantes, Destin y Svoboda (2018) señalan que la carga financiera producida por los costos de la universidad podría relacionarse con el proceso cognitivo de los estudiantes (Montalto et al. 2019; Cadaret y Bennett, 2019; Britt et al. 2017; Robb, 2017; Fosnacht y Calderone, 2017; Jones et al. 2020) y tener un determinado impacto cuando piensan en aquello. A esto se suma una correlación significativa entre préstamos estudiantiles, monto del préstamo y la graduación, lo que sugiere un impacto aparente en este indicador de logro académico (Destin y Svoboda, 2018; Coria y Hoffman, 2016; Sinchi y Gomez, 2018; Carvajal y Cervantes, 2018).

Del mismo modo, las condiciones económicas de los estudiantes tienen influencia en sus resultados académicos. Sin embargo, el impacto de las ayudas financieras sobre la persistencia es inconcluso, ya que, mientras no aparece un efecto aparente en universidades españolas, según Berlanga et al. (2017); Díaz (2017:2) sobre el caso chileno, concluye que "alumnos con ayudas financieras tienen, en promedio, una probabilidad mayor de persistencia en el 
año siguiente".

Otros estudios sugieren que se produce un impacto aparentemente positivo en los indicadores de deserción y graduación según parámetros de estudiantes becados en una universidad italiana (Agasisti y Murtinu, 2016). Se denota además un impacto significativo de una subvención en la probabilidad de graduación (Gershenfeld, Zhan \&; Hood, 2019), al igual que apuntar a que las ayudas financieras ayudarían a combatir el abandono universitario en Colombia (Guerrero y Soto, 2019).

\subsection{Ayudas financieras e inclusión: oportunidades de estudiantes de bajos ingresos, minorías y en situación de discapacidad}

Desde una mirada socioeconómica, los resultados de Coria y Hoffman (2016) constatan que las ayudas financieras reducen los efectos negativos de estudiantes con bajos ingresos, inclusive podría existir una relación proporcional de carácter directo con el monto de la ayuda financiera. En contraste, los resultados de Berlanga et al. (2016) sugieren que la relación entre las variables en estudio tiene un mayor impacto en la tasa de aprobación de cursos que en la persistencia. A esto es necesario sumar el estrés financiero, la angustia financiera y el impacto al bienestar del alumno, antes mencionado, que causan préstamos y créditos universitarios grandes (Montalto et al. 2019; Cadaret y Bennett, 2019; Britt et al. 2017; Robb, 2017; Fosnacht y Calderone, 2017; Jones et al. 2020).

A ello, cabe agregar que Wei et al. (2018) consignan en sus resultados que los préstamos adquiridos por estudiantes chinos se asocian en alguna medida con el desempeño de sus calificaciones, pero son poco significativos. Constatan además que las ayudas financieras basadas en la necesidad, como las becas, no tienen efectos significativos en los estudiantes (Wei et al. 2018). Esto se contrapone a los resultados de la mayoría de los estudios del corpus y podría explicarse por algunas variables de contexto en la educación universitaria de China, como, por ejemplo, el elevado valor de los aranceles, la proporción del monto de aranceles respecto del total de ingresos familiares y la propia cultura.

Con base en los resultados de las investigaciones del corpus, es posible constatar que existe una relación entre las ayudas financieras y el rendimiento académico, donde el grado de asociación entre variables depende de la naturaleza y tipo de ayuda financiera y también de otros factores de contexto que inciden en el impacto potencial en los indicadores de éxito estudiantil.

Además, se destacan casos de inclusión de minorías afroamericanas y latinas en Estados Unidos (Gipson et al. 2018; Carales, 2020), al igual que estudiantes mayas en México (Peniche y Ramón, 2018); grupos vulnerables (Sandoval et al. 2018); y estudiantes en situación de discapacidad (Paz, 2020), que apuntan a la necesidad de sensibilizar a la comunidad universitaria para crear mayores vínculos sociales entre estos estudiantes para fomentar la persistencia y mejorar las políticas públicas de inclusión, ya que las ayudas financieras son solo un aspecto necesario para mejorar la accesibilidad, especialmente en la caso de la discapacidad.

Los resultados de Sanchez, Lowman y Hill (2018) proponen, a partir de un estudio a 298 estudiantes de una 
universidad pública que participaron de un programa de preparación temprana para ingresar a la universidad llamado GEAR UP, que, pese a obtener puntajes más bajos en exámenes estandarizados para ingresar a la universidad en Estados Unidos (ACT), tuvieron un rendimiento y persistencia igual al grupo de control compuesto por 1841 estudiantes. Además, este estudio detectó que el GPA junto a los créditos obtenidos podrían ser predictores de persistencia, y que los estudiantes de este programa tenían mayor probabilidad de ser hispanos o latinos y menor probabilidad de ser de áreas urbanas del estado (Sanchez, Lowman y Hill, 2018).

Otros estudios abordan factores predictores de resultados académicos en participantes de programas para estudiantes latinos migrantes de primera generación, sugiriendo que el rendimiento en la escuela secundaria podría ser un predictor significativo del rendimiento en la universidad, al igual que vivir en el campus, y que los inconvenientes financieros, junto a las responsabilidades familiares y laborales aumentarían las probabilidades de abandonar la universidad (Mendez \& Bauman, 2018).

\subsection{Variables relacionadas que impactan el desempeño estudiantil}

En el análisis del corpus surge una serie de variables que podrían tener un impacto en el desempeño de los estudiantes en la universidad, siendo las de mayor recurrencia aquellas relacionadas con las condiciones y variables personales y de estudio, lo que sugiere que por sí solas o de forma combinada incluso podrían predecir el nivel de logro de los alumnos.

Según lo que plantean Doyle et al. (2019) los programas de becas orientados a comunidades de aprendizaje podrían tener un impacto en el logro académico de los estudiantes. Esto se refleja particularmente, en la retención, que es una construcción compleja en donde intervienen múltiples variables, más allá del GPA o PGC (Promedio General de Calificaciones) dentro del aula, ya que el desarrollo de la comunidad lograría ser un espacio propicio para el aprendizaje, al incentivarlos a vivir en un espacio universitario como primera opción.

Algunos estudios abordan elementos relevantes que podrían sugerir relaciones entre variables como el impacto del tiempo de estudio en el éxito estudiantil y la persistencia y graduación, sugiriendo que los estudiantes que asignan al estudio una parte de su tiempo de ocio durante el fin de semana tendrían mejores resultados, lo cual es coherente con la necesidad de tener una autoestima favorable para valorar positivamente un sacrificio que contribuya a sus logros futuros en el ámbito profesional (Escobar et al. 2019).

En la misma línea, pareciera que las condiciones de contexto de los estudiantes tiene cierta implicancia en sus logros académicos, donde el estudio individual, el tiempo dedicado al estudio, el compromiso de la institución, el contexto familiar, la ayuda financiera y el esfuerzo parecieran ser variables que promoverían la persistencia de los alumnos (Gipson, et al. 2018) junto a la importancia que tienen las variables psicosociales como la eficacia académica, resolución de problemas y conexión con la institución en la predicción de variables de éxito estudiantil, con un efecto potencial indirecto en la retención de los alumnos 
(Sass et al. 2018).

En esta línea, von Hippel \& Hofflinger (2020) señalan que las calificaciones universitarias y las ayudas financiera aportan a mejorar la predicción de la persistencia de los alumnos y que las intervenciones a partir de programas de éxito estudiantil en las instituciones deben estar orientados a estudiantes de mayor riesgo, para mejorar su efectividad. A su vez Burns et al. (2019) constatan que los programas de crédito universitario temprano tendrían un impacto positivo y significativo en la reducción del tiempo para graduarse. A ello, Cadaret y Bennett (2019) suman que la angustia general y GPA más bajos están relacionados con el estrés financiero de los estudiantes universitarios.

Finalmente, es posible detectar una serie de variables de contexto de los estudiantes que podrían incidir y tener un efecto potencial en sus resultados académicos e indicadores de éxito estudiantil, siendo aparentemente las de mayor relevancia aquellas relacionadas con las relaciones familiares, el tiempo de estudio, factores étnicos, la residencia geográfica, el estrés financiero y ciertas variables psicosociales, que podrían abordarse a partir de programas de éxito estudiantil de las instituciones que estén focalizados en los grupos de mayor riesgo.

\section{Conclusiones}

El aumento significativo de la matrícula de estudiantes de bajos ingresos a la educación superior universitaria ha generado gran interés en la comunidad científica para investigar el impacto que genera la condición más desfavorable de dichos alumnos en sus resultados académicos. Se considera que, pese al mayor acceso a través de políticas de financiamiento basadas en becas y préstamos, las brechas de conocimiento junto a otros factores han impedido lograr las tasas de retención y graduación esperadas.

En esta línea, el presente artículo ha tomado una muestra de 70 artículos sobre el desempeño académico con ayudas financieras, entre los años 2015 y 2021, para identificar los indicadores de éxito estudiantil y establecer relaciones entre tipos de ayuda, desempeño y variables de contexto.

La literatura ha analizado los efectos que tienen estas ayudas financieras en el desempeño de los estudiantes universitarios, detectando que aquellas basadas en la necesidad como las becas, tienen un impacto positivo en los indicadores de rendimiento y graduación. Ello mantiene una magnitud del efecto que depende de otras variables relacionadas, como la caracterización de las instituciones y estudiantes, junto a las variables de contexto. Estos resultados son mixtos en la persistencia, en donde hay casos variados que evidencian la heterogeneidad de las ayudas financieras al ser condicionadas por una serie de factores sociales y contextuales que las apremian.

La literatura sugiere que las disciplinas con impactos más significativos podrían ser ingeniería y algunas especialidades STEM; no obstante, es interesante notar que la escasez de literatura al respecto, por lo cual estos resultados se consideran inconclusos y se destaca la necesidad de indagar en estos efectos.

Por otra parte, las ayudas financieras basadas en préstamos tienen un efecto mucho más heterogéneo $\mathrm{y}$ poco consistente, donde pareciera 
que aquellos tipos de préstamos más tradicionales tendrían un efecto negativo y aquellos diseñados en programas más focalizados con orientación a perfiles más específicos de estudiantes tendrían un impacto positivo. Además, cabe destacar que, los préstamos y créditos financieros ejercen una presión sobre la mente y el bienestar general del estudiante que afectan su capacidad de lograr sus metas.

En ambos casos, tanto de ayudas financieras basadas en becas $o$ préstamos, es posible visualizar en los efectos de las ayudas financieras sobre el desempeño estudiantil una serie de variables adicionales y de contexto, como las relaciones familiares, el tiempo de estudio, factores étnicos, la residencia geográfica, el estrés financiero y ciertas variables psicosociales que podrían intervenir e incluso predecir los indicadores de logro.

En torno a la inclusión, tanto para estudiantes de bajos ingresos, establecida como una categoría de estudio destacada para las ayudas financieras, como casos de minorías y estudiantes en situación de discapacidad, la literatura indica que la falta de comunidad entre estudiantes es un factor importante en cuanto a persistencia universitaria y que las ayudas financieras son solo parte de la discusión sobre accesibilidad universitaria.

En este escenario, la presente investigación permite consolidar los efectos detectados en la literatura que se generan a partir de los mecanismos de ayudas financieras para estudiantes, lo que podría postularse con un punto de partida para contribuir al desarrollo de futuras investigaciones que permitan detectar con mayor claridad la significancia de los préstamos en el desempeño académico, las disciplinas donde el impacto es más consistente y vincular los efectos de las ayudas financieras con los perfiles y características de estudiantes e instituciones, desagregando el impacto, por ejemplo, a partir del género, naturaleza y orientación de los programas de financiamiento para la educación superior, y aportando de este modo a la selección de programas efectivos y a la toma de decisiones de la política pública.

\section{Referencias Bibliográficas}

Agasisti, T. y Murtinu, S. (2016).Grants in Italian university: a look at the heterogeneity of their impact on students' performances. Studies in Higher Education, 41(6), 1106-1132. https://bit.ly/3domMOC

Angarita, J. S., Solano, J. D., \& Contreras, O. A. (2017). Los matices del proceso enseñanza-aprendizaje en un estudiante de maestría con discapacidad visual. Inclusión \& Desarrollo, 4(1), 24-34. https://bit. Iy/2U6nWre

Berlanga, V., Figuera, P., y Perez-Escoda, N. (2016). Academic Performance and Persistence of Study Bursary Holders [Desempeño académico y persistencia de tenientes de subsidios de estudio]. Revista de Cercetare si Interventie Sociala, 54, 23-35. https://dialnet.unirioja.es/ servlet/articulo?codigo $=5655724$

Berlanga-Silvente, V., Guardia-Olmos, J., Figuera-Gazo, P., y Pons-Fanals, E. (2017). Salary scholarships as a factor associated with improved academic performance, Advances and Applications in Statistics. Advances and Applications in Statistics, 50(4), 329-348. https:// dx.doi.org/10.17654/AS050040329 
Gallardo Canales, Rodrigo; Pinto Inostroza, Mario; Aguirre Boza, Álvaro

Desempeño Académico de Estudiantes Universitarios con Ayudas Financieras: Una

Revisión de Literatura

Bettinger, E., Gurantz, O., Kawano, L., Sacerdote, B., \& Stevens, M. (2019). The Long-Run Impacts of Financial Aid: Evidence from California's Cal Grant. American Economic Journal: Economic Policy, 11(1), 64-94. $\quad$ https://www.aeaweb.org/ articles?id=10.1257/pol.20170466

Britt, S.L., Ammerman, D.A., Barrett, S.F., \& Jones, S. (2017). Student loans, financial stress, and college student retention. Journal of Student Financial Aid, 3. http://publications. nasfaa.org/isfa/vol47/iss $1 / 3$.

Broton, K. M. (2019). Rethinking the cooling out hypothesis for the 21st century: The impact of financial aid on students' educational goals. Community College Review, 47(1), 79-104.

Burns, K., Ellegood, W., Bracy, J. M., Duncan, M., y Sweeney, D., (2019). Early College Credit Programs Positively Impact Student Success. Journal of Advanced Academics, 30(1). https://doi. org/10.1177/1932202X18801274

Cadaret, M. C. \& Bennett, S. B. (2019). College Students' Reported Financial Stress and Its Relationship to Psychological Distress. Journal of College Counseling, 22(3). https:// doi.org/10.1002/jocc.12139

Carales, V. D. (2020). Examining Educational Attainment Outcomes: A Focus on Latina/o Community College Students. Community College Review, 48(2). https://doi. org/10.1177/0091552120903087

Carpenter, D. M., Kaka, S. J., Tygret, J.A. y Cathcart, K. (2018). Testing the Efficacy of a Scholarship Program for Single Parent, Post-Freshmen, Full Time Undergraduates. Research in Higher Education, 59(2), 1-24. https://doi.org/10.1007/s11162-0179456-0
Carvajal, R. A., \& Cervantes, C. T. (2018). Aproximaciones a la deserción universitaria en Chile. Educação e Pesquisa, 44. https://www.scielo.br/j/ ep/a/885ryWsMG8jfw4SnfSwpytN/?! ang=es\&format $=p d f$

Catalán, X., \& Santelices, M. V. (2014). Rendimiento académico de estudiantes de distinto nivel socioeconómico en universidades: el caso de la Pontificia Universidad Católica de Chile. Calidad en la educación, (40), 21-52. https:// dx.doi.org/10.4067/S071845652014000100002

Chen, J., \& Hossler, D. (2017). The effects of financial aid on college success of two-year beginning nontraditional students. Research in Higher Education, 58(1), 40-76. https://link. springer.com/article/10.1007/s11162016-9416-0

Considine, G. \& Zappala, G. (2002). The influence of social and economic disadvantage in the academic performance of school students in Australia. Journal of Sociology. The Australian Sociological Association. 38(2), 129-148. https://journals. sagepub.com/doi/10.1177/14407830 2128756543

Coria, E. \& Hoffman, J.L (2016) Financial aid tipping points: an analysis of aid and academic achievement at a California community college. Community College Journal of Research and Practice, 40(2), 160170, https://doi.org/10.1080/1066892 6.2014.993441

Correa, J. B. (2013). Educación superior e inclusión social: una perspectiva desde las instituciones universitarias salesianas. Educación y futuro: revista de investigación aplicada $y$ experiencias educativas, (28), 201-222. https://dialnet.unirioja.es/ servlet/articulo?codigo $=4152280$ 
Destin, M., y Svoboda, R.C. (2018). Costs on the Mind: The Influence of the Financial Burden of College on Academic Performance and Cognitive Functioning. Research in Higher Education. https://doi. org/10.1007/s11162-017-9469-8

Díaz, R. (2017). El impacto del tipo de financiamiento sobre la probabilidad de retención de primer año en la educación superior: el caso de la universidad de Chile. Congreso CLABES VII, Córdoba, Argentina. https://revistas.utp.ac.pa/index.php/ clabes/article/view/1586

Doyle, J., Wheelis, M., Thompson L. \& Strietzel, J. (2019). The Impact of Living-Learning Program Scholarships on Scholarship Recipients. Journal of Student Affairs Research and Practice, 56(2), 221234, https://doi.org/10.1080/1949659 $\underline{1.2018 .1474763}$

Escobar, E., Torres, D., Layedra, W. y Zhinin, W. (2019). La influencia de la concesión de becas y la capacidad de aprendizaje de los estudiantes sobre la cantidad de personas con mayor formación académica. Revista Dilemas Contemporáneos Educación, Política y Valores, VII(1). https://bit.ly/2UDZE8a

Fernández-Martín, T., Solís-Salazar, M., Hernández-Jiménez, M. T., \& Moreira-Mora, T. E. (2019). Un análisis multinomial y predictivo de los factores asociados a la deserción universitaria. Revista Electrónica Educare, 23(1), 73-97. https://bit. Iy/3qv1jZM

Fosnacht, K., \& Calderone, S. M. (2017). Undergraduate Financial Stress, Financial Self-Efficacy, and Major Choice: A Multi-Institutional Study. Journal of Financial Therapy, 8(1), $7 . \quad$ https://doi.org/10.4148/1944$\underline{9771.1129}$
Gershenfeld, S., Zhan, M., y Ward Hood, D. (2019). The Impact of a Promise: A Loan Replacement Grant, Low-Income Students, and College Graduation. The Review of Higher Education, 42(3), 1073-1100. https:// doi.org/10.1353/rhe.2019.0030

Gipson, J., Mitchell Jr. D. \& McLean, C. (2017). An Investigation of High Achieving African-American Students Attending Community Colleges: A Mixed Methods Research Study. Community College Journal of Research and Practice, 42(4), 289301. https://doi.org/10.1080/1066892 $\underline{6.2017 .1299652}$

Goldhaber, D., Long, M. C., Person, A. E., Rooklyn, J., \& Gratz, T. (2019). Sign me up: The factors predicting students' enrollment in an early-commitment scholarship program. AERA Open, 5(2), https://journals.sagepub.com/doi/ full/10.1177/2332858419857703

Graziosi, G., Sneyers, E., Agasisti T. \& Witte K. (2020). Can grants affect student performance? Evidence from five Italian universities. Journal of Higher Education Policy and Management, 43(1). https://bit. Iy/2U7JWII

Guerrero, S. C., \& Soto Arango, D. E. (2019). La política educativa en torno a la masificación de la educación superior y su relación con el abandono universitario en Colombia. Revista historia de la educación latinoamericana, 21(32), 109-136.

Herbaut, E., \& Geven, K. M. (2019). What works to reduce inequalities in higher education? A systematic review of the (quasi-)experimental literature on outreach and financial aid. Research in Social Stratification and Mobility, $65 . \quad$ https://www. sciencedirect.com/science/article/ 
Gallardo Canales, Rodrigo; Pinto Inostroza, Mario; Aguirre Boza, Álvaro

Desempeño Académico de Estudiantes Universitarios con Ayudas Financieras: Una

Revisión de Literatura

\section{abs/pii/S0276562419300770}

Huang, W., Li, F., Liao, X., y Hu, P. (2018).

More money, better performance?

The effects of student loans and need-based grants in China's higher education. China Economic Review, Elsevier, 51(C), 208-227. https:// ideas.repec.org/a/eee/chieco/ v51y2018icp208-227.html

Jones, T. R., Kreisman, D., Rubenstein, R., Searcy, C., \& Bhatt, R. (2020). The Effects of Financial Aid Loss on Persistence and Graduation: A Multi-Dimensional Regression Discontinuity Approach. IZA Discussion Papers 13849, Institute of Labor Economics (IZA). https://ideas. repec.org/p/iza/izadps/dp13849.html

Lattuada, M. (2017). Deserción y retención en las unidades académicas de educación superior. Una aproximación a las causas, instrumentos y estrategias que contribuyen a conocer y morigerar su impacto. Debate Universitario, 5(10), 100-113.

Marx, B. M. and Turner, L. J. (2019): Student Loan Nudges: Experimental Evidence on Borrowing and Educational Attainment. American Economic Journal: Economic Policy, 11(2), 108-41. https://doi. org/10.1257/pol.20180279

Melguizo, T., Sanchez, F., y Velasco, T. (2016). Credit for Low-Income Students and Access to and Academic Performance in Higher Education in Colombia: A Regression Discontinuity Approach. World Development, 80, 61-77. https://dx.doi.org/10.1016/j. worlddev.2015.11.018

Mendez, J. I., y Bauman, S. (2018). From Migrant Farmworkers to First Generation Latina/o Students: Factors Predicting College Outcomes for Students Participating in the College Assistance Migrant Program. The
Review of Higher Education, 42(1), 173-208. https://doi.org/10.1353/ rhe.2018.0037

Montalto, C. P., Phillips, E. L., McDaniel, A., \& Baker, A. R. (2019). College student financial wellness: Student loans and beyond. Journal of Family and Economic Issues, 40(1), 3-21. https://link.springer.com/ article/10.1007/s10834-018-9593-4

Moreira, G. D. O., Passeri, S., Velho, P. E. N. F., Ferraresi, F., Appenzeller, S., \& Amaral, E. (2019). The academic performance of scholarship students during medical school. Revista Brasileira de Educação Médica, 43(3), 163-169. https://bit. Iy/36SVEUt

Ononye, L., \& Bong, S. (2018). The Study of the Effectiveness of Scholarship Grant Program on Low-Income Engineering Technology Students. Journal of STEM Education: Innovations and Research, 18(5), 26-31. https://eric. ed.gov/?id=EJ1170100

Paz, E. (2020). Revisión sistemática: inclusión educativa de estudiantes universitarios en situación de discapacidad en América Latina. Estudios pedagógicos (Valdivia), 46(1), 413-429. https://www.scielo.cl/scielo. php?script=sci arttext\&pid=S0718$07052020000100413 \&$ lang $=p t$

Robb, C. A. (2017). College student financial stress: Are the kids alright? Journal of Family and Economic Issues, 38(4), 514-527. https:// link.springer.com/article/10.1007/ s10834-017-9527-6

Rodriguez Tapia, C., Caguanco Almeida, G., Rosero Portilla, S., y Coca Freire, D. (2019). Análisis de la incidencia en el rendimiento académico de los estudiantes becados tomando en cuenta las horas de estudio y 
las calificaciones de un grupo de la Universidad Regional Autónoma de los Andes. Revista Dilemas Contemporáneos Educación, Política y Valores, VII(1). https://doi. org/10.46377/dilemas.v28i1.1666

Roksa, J., y Kinsley, P. (2019). The Role of Family Support in Facilitating Academic Success of Low-Income Students. Research in Higher Education, 1-22. https://bit. ly/3z72m5h

Salinas, D. A., Hernández, A. E. y Barboza-Palomino, M. (2017). Condición de becario y rendimiento académico en estudiantes de una universidad peruana. Revista Electrónica de Investigación Educativa, 19(4), 124-133. https://doi. org/10.24320/redie.2017.19.4.1348

Sanchez, J. E., Lowman, J., y Hill, K. A. (2018). Performance and Persistence Outcomes of GEAR UP Students: Leveling the Playing Field in Higher Education, Journal of College Student Retention: Research. Journal of College Student Retention: Research, Theory \& Practice, 20(3), 328-349. https://doi. org/10.1177/1521025116669954

Sandoval, I., Sánchez, T., Velasteguí, V., \& Naranjo, D. (2018). Factores asociados al Abandono en estudiantes de grupos vulnerables. Caso Escuela Politécnica Nacional. In Congresos CLABES. https:// revistas.utp.ac.pa/index.php/clabes/ article/view/1907/2850

Sass, D.A., Castro-Villarreal, F., Wilkerson, S., Guerra, N., y Sullivan, J. (2018). A Structural Model for Predicting Student Retention. Review of Higher Education, https:// doi.org/10.1353/rhe.2018.0035

Sinchi Nacipucha, E. R., \& Gómez Ceballos, G. P. (2018). Acceso y deserción en las universidades. Alternativas de financiamiento. ALTERIDAD. Revista de Educación, 13(2), 274-287. https://alteridad.ups.edu.ec/index. php/alteridad/article/view/2.2018.10

Stoddard, C., Urban, C., y Schmeiser, M. D. (2018). College Financing Choices and Academic Performance. Journal of Consumer Affairs, 52(3), 540-561. https://onlinelibrary.wiley.com/doi/ abs/10.1111/joca.12175

Tinto, V. (1993). Leaving College: Rethinking the Causes and Cures of Student Attrition. 2da. Ed. University of Chicago Press.

Troiano, H., y Elias, M. (2014). University access and after: explaining the social composition of degree programmes and the contrasting expectations of students, High Educ, 67, 637-654. https://doi.org/10.1007/s10734-0139670-4

von Hippel, P., and Hofflinger, A. (2017). The Data Revolution Comes to Higher Education: Identifying Students at Risk of Dropout in Chile. Journal of Higher Education Policy and Management, 43(6), 1-22 http:// dx.doi.org/10.2139/ssrn.3073912

Whatley, M., \& Clayton, A. B. (2020). Study abroad for low-income students: The relationship between need-based grant aid and access to education abroad. Journal of Student Financial Aid, 49(2), 1. https:// ir.library.louisville.edu/jsfa/vol49/ iss $2 / 1 /$

Young, E., Zhang, W., y Zhang, D. (2013). Editorial: Building a lifelong learning society. International Journal of Continuing Education and Lifelong Learning, 4(1), pp. iii-iv. https:// search.informit.org/doi/10.3316/ INFORMIT.752823232703279 


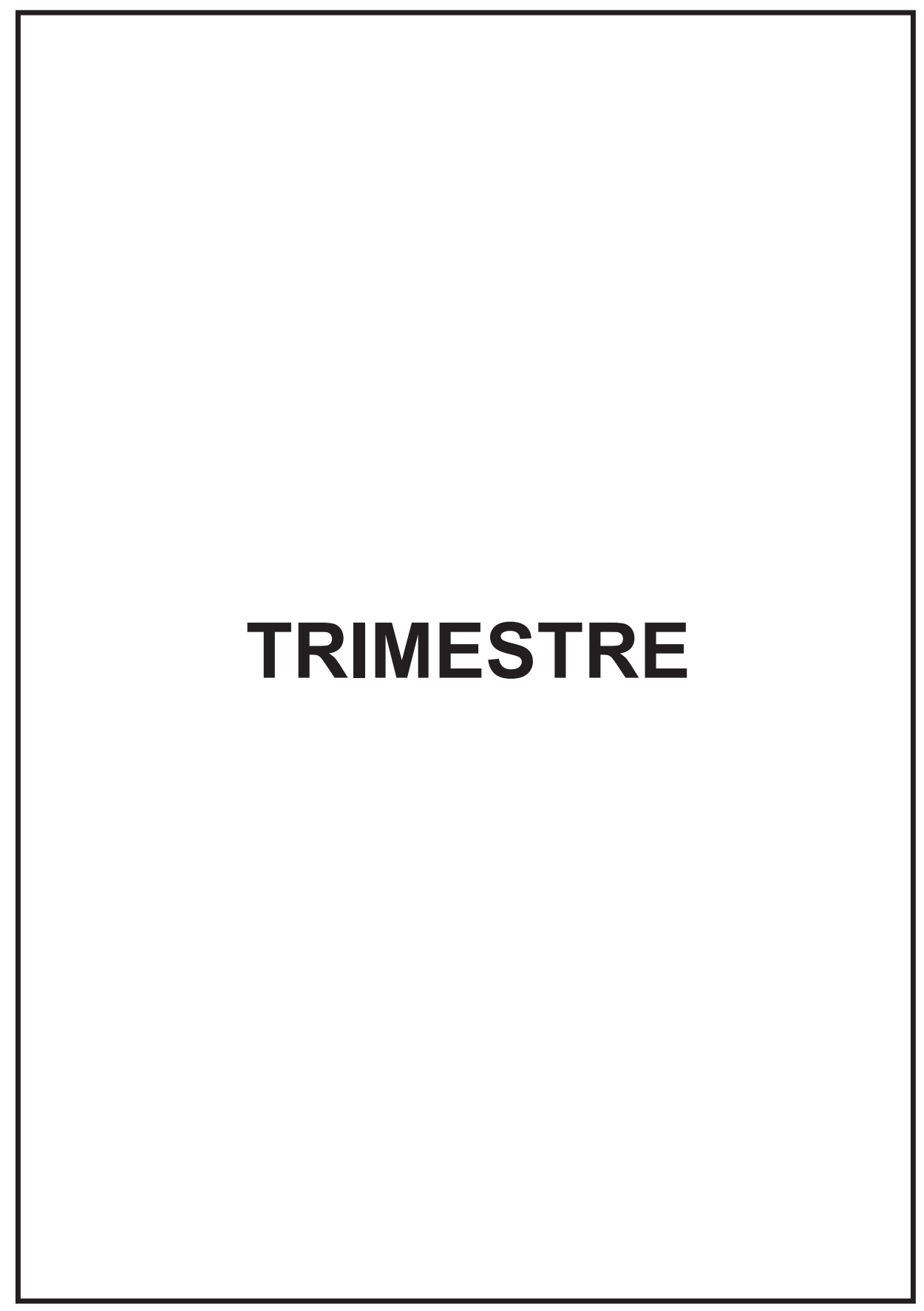

